PROCEEDINGS OF THE

AMERICAN MATHEMATICAL SOCIETY

Volume 128, Number 8, Pages 2439-2444

S 0002-9939(99)05318-6

Article electronically published on November 24, 1999

\title{
AN EXTENSION OF MULTI-VALUED CONTRACTION MAPPINGS AND FIXED POINTS
}

\author{
CHENG-KUI ZHONG, JIANG ZHU, AND PEI-HAO ZHAO
}

(Communicated by David R. Larson)

\begin{abstract}
A class of new multi-valued contraction mappings is presented. Under the new contractive conditions, some fixed point theorems for multivalued self-mappings and nonself-mappings are proved.
\end{abstract}

\section{INTRODUCTION}

The well-known Banach contraction principle has extensive applications in many fields of mathematics and applied mathematics. For example, it has become a vigorous tool for studying nonlinear Volterra integral equations, nonlinear integraldifferential equations and nonlinear functional differential equations in Banach space. Because of its importance for mathematical theory, it has been generalized to multi-valued mappings by many authors. For some of these results, one can refer to Caristi [1],Nadler [2, Yi-Zhao [3] and Sehgal-Smithson [4]. However, the contractive coefficients in all these generalizations are constant. In this note, we advance a class of new multi-valued contractive conditions which are essentially different from the earlier ones. Under the new contractive conditions, some fixed point theorems for multi-valued self-mappings and nonself-mappings are proved. Our results are new generalizations of the Banach contraction principle, and even for single-valued mappings, these are the newest results. Moreover, our results are generalizations for the corresponding results in Nadler [2], Yi-Zhao [3] and SehgalSmithson [4]. In addition, we also give some example to show that our conditions essentially generalize the earlier contractive conditions and that our results are best to some extent.

Our results are based on the new result proved by the first author in the recent paper [5]. Throughout this paper, we denote by $(X, d)$ a complete metric space, by $C B(X)$ the set of all non-empty bounded and closed subsets of $X$ and by $C(X)$ the set of all non-empty compact subsets of $X$. For any $A, B \in C B(X)$, the Hausdorff metric is defined by

$$
H(A, B)=\max \left\{\sup _{x \in B} d(x, A), \sup _{x \in A} d(x, B)\right\}
$$

Received by the editors December 19, 1997 and, in revised form, September 28, 1998.

2000 Mathematics Subject Classification. Primary 47H10, 47H04.

This research was supprted by the National Science Foundation and Doctoral Foundation of China.

The corresponding author is Jiang Zhu. 
and we define $H_{+}(A, B)=\sup _{x \in B} d(x, A)$. For any $x, y \in X, x \neq y$, we denote by $(x, y]$ the left open and right closed segment, that is, the set of points $z \in X$ distinct from $x$ and satisfying $d(x, z)+d(z, y)=d(x, y)$.

The following two lemmas are important for our proof.

Lemma 1.1 (Zhong [5]). Let $h:[0,+\infty) \rightarrow[0,+\infty)$ be a continuous nondecreasing function such that $\int_{0}^{+\infty} \frac{d r}{1+h(r)}=+\infty$. Let $X$ be a complete metric space, $x_{0} \in X$ a given point and $f: X \rightarrow(-\infty,+\infty]$ a lower semi-continuous and bounded from below. Then, for any $\varepsilon>0$, any $y \in X$ satisfying

$$
f(y)<\inf _{x \in X} f(x)+\varepsilon
$$

and every $\lambda>0$, there exists a $z \in X$ such that $f(z) \leq f(y)$ and $d\left(z, x_{0}\right) \leq r_{0}+\bar{r}$ and for any $x \in X$,

$$
f(x) \geq f(z)-\frac{\varepsilon}{\lambda\left(1+h\left(d\left(x_{0}, z\right)\right)\right)} d(x, z),
$$

where $r_{0}=d\left(x_{0}, y\right)$ and $\bar{r}$ satisfies $\int_{r_{0}}^{r_{0}+\bar{r}} \frac{d r}{1+h(r)} \geq \lambda$.

From Lemma 1.1, we can get the following Lemma 1.2 which is a generalization of the well-known Caristi's [1] fixed point theorem.

Lemma 1.2. Let $(X, d)$ be a complete metric space and $x_{0} \in X$ a given point. Let $\phi$ be a lower semi-continuous function from $X$ to $[0,+\infty)$ and $h$ be as in Lemma 1.1. Let $F$ be a self-mapping of $X$ such that, for all $x \in X$,

$$
\frac{d(x, F(x))}{1+h\left(d\left(x_{0}, x\right)\right)} \leq \phi(x)-\phi(F(x)) .
$$

Then $F$ has a fixed point.

Proof. Applying Lemma 1.1 to the function $\phi$ with $\varepsilon=1 / 2$ and $\lambda=1$, we know that there exists $z_{\varepsilon} \in X$ such that

$$
\phi(x) \geq \phi\left(z_{\varepsilon}\right)-\frac{\varepsilon}{1+h\left(d\left(x_{0}, z_{\varepsilon}\right)\right)} d\left(x, z_{\varepsilon}\right), \quad \forall x \in X .
$$

Taking $F\left(z_{\varepsilon}\right)$ instead of $x$ in (2) and $z_{\varepsilon}$ instead of $x$ in (11) we get

$$
\frac{1}{1+h\left(d\left(x_{0}, z_{\varepsilon}\right)\right)} d\left(z_{\varepsilon}, F\left(z_{\varepsilon}\right)\right) \leq \phi\left(z_{\varepsilon}\right)-\phi\left(F\left(z_{\varepsilon}\right)\right) \leq \frac{\varepsilon}{1+h\left(d\left(x_{0}, z_{\varepsilon}\right)\right)} d\left(z_{\varepsilon}, F\left(z_{\varepsilon}\right)\right) .
$$

This implies $z_{\varepsilon}=F\left(z_{\varepsilon}\right)$ and completes the proof.

\section{FIXED POINTS}

We recall that a multi-valued mapping $F: X \rightarrow C B(X)$ is upper semi-continuous, if for any $x_{0} \in X$ and any $\varepsilon>0$, there exists $\eta=\eta\left(x_{0}, \varepsilon\right)>0$ such that $H_{+}\left(F\left(x_{0}\right), F(x)\right)<\varepsilon$, whenever $d\left(x_{0}, x\right)<\eta$.

Theorem 2.1. Let $F: X \rightarrow C B(X)$ be a multi-valued and upper semi-continuous mapping, $x_{0} \in X$ a given point and $\sigma \in(0,1]$ a constant. Let $h:[0,+\infty) \rightarrow[0,+\infty)$ be a continuous nondecreasing function satisfying

$$
\int_{0}^{+\infty} \frac{d r}{1+h(r)}=+\infty
$$


such that for any $x \in X$, if $x \notin F(x)$, then for any $y \in F(x)$,

$$
H_{+}(F(y), F(x)) \leq\left(1-\frac{\sigma}{1+h\left(d\left(x_{0}, x\right)\right)}\right) d(x, y) .
$$

Then $F$ has a fixed point.

Proof. Let $f: X \rightarrow[0,+\infty)$ be defined by $f(x)=d(x, F(x))$. We first prove that $f$ is lower semi-continuous. Suppose that $\left\{x_{n}\right\}$ converges to $x$; then for any $z \in F\left(x_{n}\right)$, we have

$$
\begin{aligned}
f(x) & =d(x, F(x)) \leq d\left(x, x_{n}\right)+d\left(x_{n}, z\right)+d(z, F(x)) \\
& \leq d\left(x, x_{n}\right)+d\left(x_{n}, z\right)+H_{+}\left(F(x), F\left(x_{n}\right)\right) .
\end{aligned}
$$

Since $z \in F\left(x_{n}\right)$ is arbitrary, we have

$$
f(x) \leq d\left(x, x_{n}\right)+d\left(x_{n}, F\left(x_{n}\right)\right)+H_{+}\left(F(x), F\left(x_{n}\right)\right) .
$$

It follows from the upper semi-continuity of $F$ that $f(x) \leq \liminf _{n \rightarrow \infty} f\left(x_{n}\right)$. Hence, $f$ is lower semi-continuous. By applying Lemma 1.1 with $\varepsilon=\sigma / 2$ and $\lambda=1$, we get some $z_{\varepsilon} \in X$ such that for any $x \in X$

$$
d(x, F(x)) \geq d\left(z_{\varepsilon}, F\left(z_{\varepsilon}\right)\right)-\frac{\sigma}{2\left(1+h\left(d\left(x_{0}, z_{\varepsilon}\right)\right)\right)} d\left(x, z_{\varepsilon}\right) .
$$

If $z_{\varepsilon} \notin F\left(z_{\varepsilon}\right)$, by (4) we know that for any $y \in F\left(z_{\varepsilon}\right)$ we have

$$
H_{+}\left(F(y), F\left(z_{\varepsilon}\right)\right) \leq\left(1-\frac{\sigma}{1+h\left(d\left(x_{0}, z_{\varepsilon}\right)\right)}\right) d\left(z_{\varepsilon}, y\right) .
$$

Taking $y$ instead of $x$ in (5) and combining (6) we get

$$
\begin{gathered}
d\left(z_{\varepsilon}, F\left(z_{\varepsilon}\right)\right) \leq d(y, F(y))+\frac{\sigma}{2\left(1+h\left(d\left(x_{0}, z_{\varepsilon}\right)\right)\right)} d\left(y, z_{\varepsilon}\right) \\
\leq H_{+}\left(F(y), F\left(z_{\varepsilon}\right)\right)+\frac{\sigma}{2\left(1+h\left(d\left(x_{0}, z_{\varepsilon}\right)\right)\right)} d\left(y, z_{\varepsilon}\right) \\
\leq\left(1-\frac{\sigma}{1+h\left(d\left(x_{0}, z_{\varepsilon}\right)\right)}\right) d\left(z_{\varepsilon}, y\right)+\frac{\sigma}{2\left(1+h\left(d\left(x_{0}, z_{\varepsilon}\right)\right)\right)} d\left(y, z_{\varepsilon}\right) \\
=\left(1-\frac{\sigma}{2\left(1+h\left(d\left(x_{0}, z_{\varepsilon}\right)\right)\right)}\right) d\left(y, z_{\varepsilon}\right) .
\end{gathered}
$$

This implies that

$$
d\left(z_{\varepsilon}, F\left(z_{\varepsilon}\right)\right) \leq\left(1-\frac{\sigma}{2\left(1+h\left(d\left(x_{0}, z_{\varepsilon}\right)\right)\right)}\right) d\left(z_{\varepsilon}, F\left(z_{\varepsilon}\right)\right) .
$$

This is impossible. Therefore, $z_{\varepsilon} \in F\left(z_{\varepsilon}\right)$. The proof is completed.

In the following, we shall give some examples to show that contractive condition (41) is an essential generalization of the earlier Banach type contractive condition and that (4) is best to some extent. For the sake of succinctness, we give examples involving single valued functions only.

Example 2.2. Let $X=\{(x, y) \in R \times R: 0 \leq y \leq x\}$ and the metric $|\cdot|$ be defined by $|(x, y)-(u, v)|=\max \{|x-u|,|v-y|\}$ for any $(x, y),(u, v) \in X$. Let $h:[0,+\infty) \rightarrow[0,+\infty)$ be as in Theorem 2.1 and $F: X \rightarrow X$ be a mapping defined by $F(x, y)=\left(x,\left(1-\frac{\sigma}{1+h(|(x, y)|)}\right) y\right)$, where $0<\sigma<1$ is a constant and $|(x, y)|=$ $|(0,0)-(x, y)|$. We have the following assertions.

(a) $F$ is not a Banach contraction mapping. In fact, we take $(x, y),(u, y) \in X$ such that $|x-u|>2 \sigma y$. Then, we have

$$
|F((x, y))-F((u, y))|=|x-u|=|(x, y)-(u, y)| .
$$

This shows that $F$ is not a Banach contraction mapping. 
(b) $F$ satisfies the condition of Theorem 2.1. In fact, for any $(x, y) \in X$,

$$
\begin{gathered}
\left|F^{2}((x, y))-F((x, y))\right|=\left|\left(x,\left(1-\frac{\sigma}{1+h(x)}\right)^{2} y\right)-\left(x,\left(1-\frac{\sigma}{1+h(x)}\right) y\right)\right| \\
=\left(1-\frac{\sigma}{1+h(x)}\right) \frac{\sigma}{1+h(x)} y=\left(1-\frac{\sigma}{1+h(x)}\right)|(x, y)-F((x, y))| .
\end{gathered}
$$

This shows that contractive condition (4) is satisfied. In addition, $F$ is, clearly, continuous and any $(x, 0) \in X$ is a fixed point of $F$.

From (a) and (b) we can see that Theorem 2.1 essentially generalizes the Banach contraction principle and Nadler's multi-valued contraction mapping theorems.

Example 2.3. Let $X=[0,+\infty)$ with the usual Euclidean metric, and let $T: X \rightarrow$ $X$ be defined by $T(x)=\sqrt{x^{2}+1}$. Then

$$
\begin{aligned}
& \left|T^{2} x-T x\right|=|T x-x| \frac{\sqrt{x^{2}+1}+x}{\sqrt{x^{2}+2}+\sqrt{x^{2}+1}} \\
& \quad \leq|T x-x|\left|1-\frac{1}{4 x^{2}+(\sqrt{2}+1) x+3}\right| .
\end{aligned}
$$

Let $h(r)=4 r^{2}+(\sqrt{2}+1) r+2$ and $x_{0}=0$; then $h(r)$ is a continuous nondecreasing function and $d\left(x_{0}, x\right)=x$. From (7) we have

$$
\left|T^{2} x-T x\right| \leq\left(1-\frac{1}{1+h\left(d\left(x_{0}, x\right)\right)}\right)|T x-x| .
$$

That is, $T$ and $h$ satisfy (4). But $T$ has no fixed point and

$$
\int_{0}^{+\infty} \frac{d r}{1+h(r)}=\int_{0}^{+\infty} \frac{d r}{4 r^{2}+(\sqrt{2}+1) r+3}<+\infty .
$$

This shows that the condition (3) is important for Theorem 2.1.

When $F$ is a multi-valued nonself-mapping, we have the following results.

Theorem 2.4. Let $D$ be a non-empty and closed subset of $X$. Let $F: D \rightarrow C(X)$ be a multi-valued and upper semi-continuous mapping. Let $h:[0,+\infty) \rightarrow[0,+\infty)$, $x_{0} \in X, \sigma \in(0,1]$ be as in Theorem 2.1. If for any $x \in X, x \notin F(x)$, there exists $y \in F(x)$ such that $d(x, y)=d(x, F(x)),(x, y] \cap D \neq \emptyset$ and there exists $z \in(x, y] \cap D$ with

$$
H_{+}(F(z), F(x)) \leq\left(1-\frac{\sigma}{1+h\left(d\left(x_{0}, x\right)\right)}\right) d(x, z)
$$

then $F$ has a fixed point.

Proof. Let $f: X \rightarrow(-\infty,+\infty]$ be defined by $f(x)=d(x, F(x))$, if $x \in D ; f(x)=$ $+\infty$, if $x \notin D$. It is similar to the proof of Theorem 2.1 that $f$ is lower semicontinuous. By applying Lemma 1.1 with $\varepsilon=\sigma / 2$ and $\lambda=1$, we get some $z_{\varepsilon} \in X$, such that

$$
d(x, F(x)) \geq d\left(z_{\varepsilon}, F\left(z_{\varepsilon}\right)\right)-\frac{\sigma}{2\left(1+h\left(d\left(x_{0}, z_{\varepsilon}\right)\right)\right)} d\left(x, z_{\varepsilon}\right), \quad \text { for any } \quad x \in X .
$$

If $z_{\varepsilon} \notin F\left(z_{\varepsilon}\right)$, by (8) we know that there exist a $y \in F\left(z_{\varepsilon}\right)$ and a $z \in\left(z_{\varepsilon}, y\right] \cap D$ such that

$$
d\left(z_{\varepsilon}, F\left(z_{\varepsilon}\right)\right)=d\left(z_{\varepsilon}, y\right)=d\left(z_{\varepsilon}, z\right)+d(z, y)
$$

and

$$
H_{+}\left(F(z), F\left(z_{\varepsilon}\right)\right) \leq\left(1-\frac{\sigma}{1+h\left(d\left(x_{0}, z_{\varepsilon}\right)\right)}\right) d\left(z, z_{\varepsilon}\right)
$$


Taking $z$ instead of $x$ in (9) and combining (10), (11) we have

$$
\begin{gathered}
d\left(z_{\varepsilon}, F\left(z_{\varepsilon}\right)\right) \leq d(z, F(z))+\frac{\sigma}{2\left(1+h\left(d\left(x_{0}, z_{\varepsilon}\right)\right)\right)} d\left(z, z_{\varepsilon}\right) \\
\leq d(y, F(z))+d(z, y)+\frac{\sigma}{2\left(1+h\left(d\left(x_{0}, z_{\varepsilon}\right)\right)\right)} d\left(z, z_{\varepsilon}\right) \\
\leq H_{+}\left(F(z), F\left(z_{\varepsilon}\right)\right)+d\left(z_{\varepsilon}, y\right)-d\left(z_{\varepsilon}, z\right) \\
\quad+\frac{\sigma}{2\left(1+h\left(d\left(x_{0}, z_{\varepsilon}\right)\right)\right)} d\left(z, z_{\varepsilon}\right) \\
\leq-\frac{\sigma}{2\left(1+h\left(d\left(x_{0}, z_{\varepsilon}\right)\right)\right)} d\left(z, z_{\varepsilon}\right)+d\left(z_{\varepsilon}, F\left(z_{\varepsilon}\right)\right) .
\end{gathered}
$$

This is impossible. Therefore, $z_{\varepsilon} \in F\left(z_{\varepsilon}\right)$. The proof is completed.

In the following, we assume that $X$ is a Banach space and $D$ is a closed subset of $X$. Recall that a multi-valued mapping $T: D \rightarrow C B(X)$ is called nonexpansive if $H(T x, T y) \leq\|x-y\|$ for any $x, y \in D$. $T$ is said to be a weakly inward mapping if $T x \subset \overline{I_{D}(x)}$ for all $x \in D$, where $I_{D}(x)=\{z \in X: z=x+c(y-x)$ for some $y \in D$ and $c \geq 1\}$ is the inward set of $x$ relative to $D$ and $\overline{I_{D}(x)}$ is the closure of $I_{D}(x)$.

Theorem 2.5. Let $T: D \rightarrow C(X)$ be a multi-valued weakly inward mapping, $h$ : $[0,+\infty) \rightarrow[0,+\infty), \sigma \in(0,1]$ and $x_{0} \in D$ be as in Theorem 2.1. If for any $x, y \in D$

$$
H(T x, T y) \leq\left(1-\frac{\sigma}{1+h\left(d\left(x_{0}, x\right)\right)}\right) d(x, y)
$$

then $T$ has a fixed point in D.

Proof. Suppose that $T$ has no fixed point in $D$. Then for any $x \in D$ there exists a $z \in T x$, such that $0<d(x, T x)=\|x-z\|$. We choose $0<c<\sigma$ and $q(x)=$ $(\sigma-c) / 2\left(1+h\left(d\left(x_{0}, x\right)\right)\right)$; then $0<q(x)<1$. Since $T x \subset \overline{I_{D}(x)}$, there exist a $y \in D$ and $\lambda \geq 1$ such that

$$
\|z-(x+\lambda(y-x))\|<q(x)\|x-z\| .
$$

We define $k=1 / \lambda$ and $w=(1-k) x+k z$. Then we have

$$
\begin{array}{cc}
\|w-y\|<q(x) k\|x-z\|, & \|w-x\|=k\|x-z\| \\
& \text { and } \\
\|w-z\|=(1-k)\|x-z\| .
\end{array}
$$

It follows from (14) that

$$
\|x-y\| \leq\|x-w\|+\|w-y\| \leq(1+q(x))\|w-x\| .
$$

We select $a \in T x, b \in T y$ such that

$$
\|w-a\|=d(w, T x) \quad \text { and } \quad\|a-b\| \leq H(T y, T x) .
$$

Combining (14) and (16) we imply that

$$
\begin{gathered}
d(y, T y) \leq\|y-b\| \leq\|y-w\|+\|w-a\|+\|a-b\| \\
\leq\|y-w\|+\|w-z\|+H(T y, T x) \\
=\|y-w\|+\|x-z\|-k\|x-z\|+H(T y, T x) \\
<k(q(x)-1)\|x-z\|+\|x-z\|+H(T y, T x) \\
=(q(x)-1)\|w-x\|+\|x-z\|+H(T y, T x) .
\end{gathered}
$$

Note that $0<q(x)<1$ and using (15) and (12) we have

$$
\begin{gathered}
d(y, T y)<\frac{q(x)-1}{q(x)+\underset{\sigma}{\sigma}}\|x-y\|+\|x-z\| \\
+\left(1-\frac{\left.\sigma_{0}\left(x_{0}, x\right)\right)}{1+h(d(x-y \|}\|x-y\| .\right. \\
\leq d(x, T x)-\frac{c}{1+h\left(d\left(x_{0}, x\right)\right)}\|x-y\| .
\end{gathered}
$$


Define $f(x)=y$ and $\phi(x)=1 / c \cdot d(x, T x)$ for any $x \in D$. By virtue of (12) and inequality

$$
|d(x, T x)-d(y, T y)| \leq d(x, y)+H(T y, T x)
$$

we can imply that $\phi: D \rightarrow[0,+\infty)$ is continuous. By (17) we have

$$
\frac{1}{1+h\left(d\left(x_{0}, x\right)\right)} d(x, f(x)) \leq \phi(x)-\phi(f(x)) .
$$

Applying Lemma 1.2 we know that $f$ has a fixed point. However, from (13) we know that $f$ has no fixed point. This is a contradiction and shows that $T$ has a fixed point. The proof is completed.

The following simple Example 2.6 will show that the conclusion of Theorem 2.5 cannot be extended to nonexpansive multi-valued mappings, even when one imposes an additional condition, such as condition (ii) of Theorem 2.2 in Yi and Zhao [3].

Example 2.6. Let $X=R^{2}, D=\{(x, y): x=\cos \theta, y=\sin \theta, \theta \in[0,2 \pi]\}$ and $T: D \rightarrow R^{2}$ be a mapping defined by $T(x, y)=\{(\cos (\theta+\varphi), \sin (\theta+\varphi))\}$, where $\varphi \in(0, \pi / 2)$ is a given number.

Since $T$ is a self-mapping of $D, T$ is clearly weakly inward mapping. $T$ is also nonexpansive, but $T$ has no fixed point. This shows the conclusion of Theorem 2.5 does not hold for nonexpansive mappings. In addition, we note that the set

$$
\bigcup_{(x, y) \in D}\{(x, y)-T(x, y)\}=\bigcup_{\theta \in[0,2 \pi]}\{(\cos \theta-\cos (\theta+\varphi), \sin \theta-\sin (\theta+\varphi))\}
$$

is a closed set. Thus, $T$ satisfies the conditions of Theorem 2.2 in Yi and Zhao [3] as well.

The above example shows that Theorem 2.2, thereby, its random version, Theorem 3.2 in Yi and Zhao [3] does not hold.

\section{ACKNowledgments}

We are grateful to the referee for his/her careful reading.

\section{REFERENCES}

[1] J. Caristi, Fixed point theorems for mappings satisfying inwardness conditions, Trans. Amer. Math. Soc. 215 (1976), 241-251. MR 52:15132

[2] S. B. Nadler Jr., Multivalued contraction mappings, Pacific J. Math. 30 (1969), 475-487. MR 40:8035

[3] H. W. Yi and Y. C. Zhao, Fixed point theorems for weakly inward multivalued mappings and their randomizations, J. Math. Anal. Appl. 183 (1994), 613-619. MR 95j:47072

[4] V. M. Sehgal and R. E. Smithson, A fixed point theorem for weak directional contraction multifunctions, Math. Japonica, 25 (1980), 345-348. MR 81m:54081

[5] C. K. Zhong, A generalization of Ekeland's variational principle and application to the study of the relation between the weak P. S. condition and coercitvity, Nonlinear Analysis, TMA. 29 (1997), 1421-1431. MR 98j:49036

Department of Mathematics, Lanzhou University, Lanzhou 730000, People's Republic OF CHINA

Current address, J. Zhu: Department of Mathematics, Xuzhou Normal University, Xuzhou 221009, People's Republic of China

E-mail address: jiangzhu@public.xz.js.cn 